EXTENDED REPORT

\title{
A new system for the automatic estimation of endothelial cell density in donor corneas
}

\author{
A Ruggeri, E Grisan, J Jaroszewski
}

Br J Ophthalmol 2005;89:306-311. doi: 10.1136/bjo.2004.051722

See end of article for authors' affiliations

.....................

Correspondence to: Alfredo Ruggeri,

Department of Information Engineering, University of Padova, Via Gradenigo 6/a, 35131 Padova, Italy; alfredo.ruggeri@unipd.it

Accepted for publication 1 August 2004 Aims: The problem of automatic estimation of endothelial cell density from microscopy images in donor
corneas was addressed.
Methods: The spatial frequencies contained in digital endothelium images are extracted with a two Methods: The spatial frequencies contained in digital endothelium images are extracted with a two
dimension discrete Fourier transform (DFT) technique. A circular band in the DFT of the images is shown to contain the frequency information related to the cell density. An algorithm for reliably recovering this spatial frequency information and for extracting from it an estimate of endothelial cell density has been developed and implemented in a computer program. An evaluation was performed on a data set containing 100 donor corneas, by comparing automatic values with manual counts performed by three eye bank experts on two images for each cornea.

Results: The mean difference of automatic densities $v$ manual ones was 14 cells $/ \mathrm{mm}^{2}(0.9 \%)$, with a standard deviation of 119 cells $/ \mathrm{mm}^{2}(5.1 \%)$ and mean absolute difference of 92 cells $/ \mathrm{mm}^{2}(3.9 \%)$. The ANOVA based overall inter-rater reliability was 0.935 . The algorithm was also capable of identifying all non-processable images. Running times were in the order of 1-2 seconds per image.

Conclusion: A new algorithm was developed for the fully automatic estimation of endothelial cell density. The results of a clinical evaluation on 100 corneas suggest that it is capable of reliably estimating endothelium cell density in donor corneas.
M icroscopy images of donor corneal endothelium are commonly analysed at eye banks for the clinical assessment of cornea quality and suitability for transplantation. Images are usually obtained with a specular or standard optical microscope and quantitative analysis is performed by visual inspection of images. ${ }^{1}$ To determine endothelial cell density experts commonly count all the cells inside a square reticle, which frames only a small fraction of the image, by either looking through the microscope eyepiece or at a printed image. Usually only 20-30 cells fall within the reticle and from this count the endothelial cell density for the whole cornea is extrapolated. This manual procedure is quite tedious, highly subjective, and error prone.

Since the availability of digital image acquisition devices and processing techniques, several prototypes aiming at the automatic extraction of cell contours and analysis of corneal endothelium have been proposed..$^{2-13}$ However, images are often blurred and noisy and a correct recognition of the contour of the cells is hard to achieve. Hence, all these systems may be classified as semiautomatic, as they require operator interaction to guide the process and correct errors: without tedious and time consuming manual editing, none of the proposed systems is able to obtain a reliable estimation of the quantitative indexes. For this reason, most of them did not go beyond the research prototype stage and, to the best of our knowledge, none is in routine clinical use at a significant number of institutions.

By observing some regularity in the endothelial cell pattern present in the images (fig 1 ), given by the repetitive element of the difference between the dark cell bodies and the brighter intercellular space, we decided to address the problem in a simpler way-that is, in the frequency domain, and to avoid any complex contour recognition tasks. Masters first suggested the use of Fourier transform to analyse human corneal endothelial cell patterns ${ }^{14}$; later on, authors from the same group showed that the Fourier analysis may in principle provide information on the cell size and thus cell density, ${ }^{15}$ but did not provide any description of this relation or propose any methodology or technique to actually extract and use this information.

In this paper, we present a complete automatic system to estimate endothelial cell density by means of the Fourier analysis, and the results of its clinical evaluation on a data set containing 100 corneas.

\section{MATERIALS AND METHODS}

Images of corneal endothelium were acquired following the procedures normally used at Cornea Bank Berlin. Corneas were kept in hypotonic balanced salt solution (BSS) for a better microscopy visualisation of the endothelial cells by osmotic stimulation. Endothelial cell images were acquired before organ culture or after de-swelling in organ culture medium (minimum essential medium (MEM) with $2 \%$ fetal calf serum (FCS)) containing 6\% dextran 5000, in order to have a low amount of folds of the Descemet membrane and a large area of endothelial cells in focus. Only corneas with clearly visible endothelial cells after osmotic stimulation were used for this study.

The framed area was $1256 \mu \mathrm{m} \times 940 \mu \mathrm{m}$ (area size $1180640 \mu^{2}$ ) and was located in the central zone of the cornea, to avoid bias in the cell density evaluation. ${ }^{16}$ Images were acquired using an inverse phase contrast microscope (CK 40, Olympus Co, Japan) at $100 \times$ and $200 \times$ magnification $(10 \times$ and $20 \times$ at the objective) and an analogue camera (SSC-DC50AP, Sony Co, Japan), and were then digitised by a personal computer with a frame grabber board (Meteor-II, Matrox Graphics Inc, Canada) as 8 bit grey level images at PAL resolution of $768 \times 576$ pixels (see, for example, fig 1).

A spatial frequency analysis was applied to the acquired images by means of the two dimensional discrete Fourier

Abbreviations: BSS, balanced salt solution; DFT, discrete Fourier transform; FCS, fetal calf serum; MEM, minimum essential medium; ROI, region of interest 


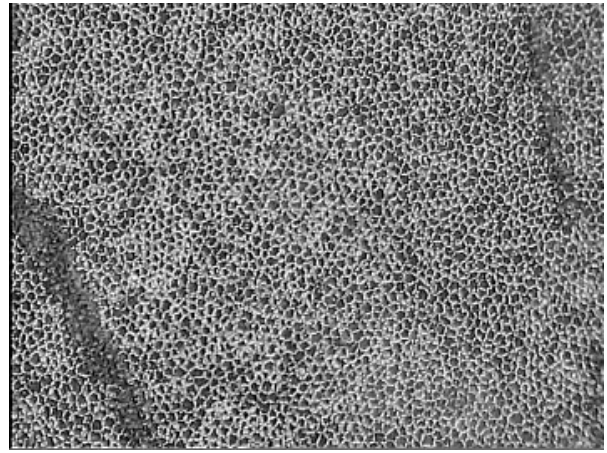

Figure 1 Image of a typical endothelium central area of a donor cornea, acquired with a $100 \times$ optical microscope with phase contrast filter.

transform (DFT). ${ }^{17}$ This mathematical technique extracts from an image the information regarding the various spatial frequencies contained-that is, about the repetitive patterns present in the image. The magnitude of these frequency components can be represented as a second image: in figure 2 , the magnitude of the DFT of the image shown in figure $l$ is displayed. Vertical and horizontal axes represent vertical and horizontal frequency components, respectively, and the magnitudes of these components are represented as grey levels (low magnitudes are darker, high magnitudes are brighter).

In figure 2, it can be seen that the resulting image shows a specific type of pattern: a circular bright band around the origin can be observed. It indicates that the magnitude of frequencies has a peak in this circular region, being the DFT image here brighter than elsewhere. This may be interpreted as the image having the frequencies inside this band as dominant frequencies. The position of the band changes according to the analysed specimen. A comparison between endothelium images from different corneas shows that the radius of this band is monotonically related to the cell density manually estimated by eye bank experts. In figure 3, two endothelium images are shown together with their DFT images: the image with higher cell density has the bright circular band with the larger radius and vice versa. Thus, the radius of the bright circular band, which represents the spatial frequency of the cells' repetitive pattern, is related to the cell density.

The presence of the circular band in the DFT images of corneal endothelium and its relation to the cell density can also be theoretically justified by the definition of the two dimensional Fourier transform and the central projection theorem. ${ }^{17}$

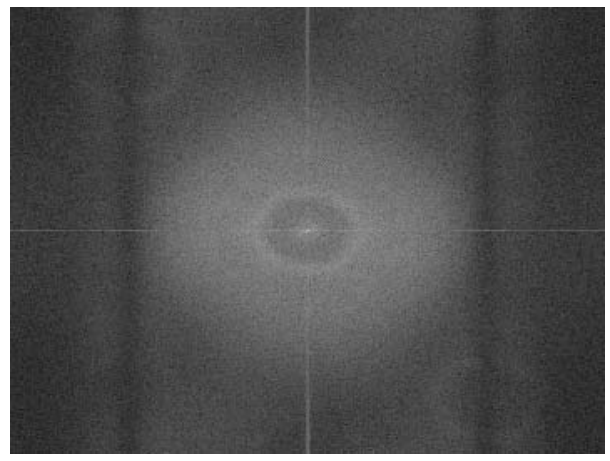

Figure 2 The frequency content (log magnitude of DFT) of the image shown in figure 1.
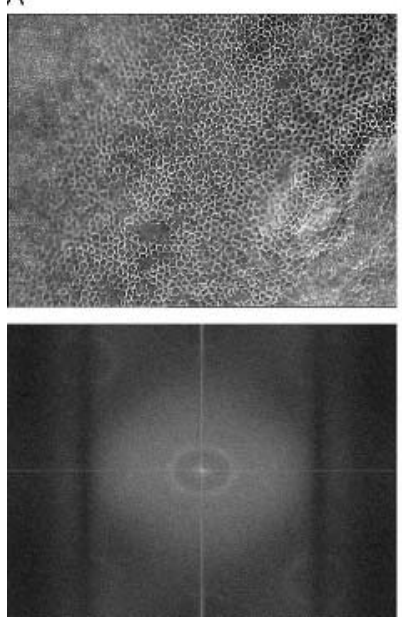

Figure 3 Top row: images of a low (left) and high (right) density corneal endothelium; bottom row: corresponding images of the frequency content (log magnitude of DFT).

\section{Frequency content extraction}

As observed in figure 2, the circular band in the DFT magnitude image is roughly circularly symmetric around the origin. The frequency content of the cell field can therefore be assumed to be approximately the same along any direction (horizontal, vertical, or diagonal) in the image. This is also confirmed by the anatomical knowledge that the corneal endothelium cell field has no directionality, and by the visual inspection of images, where no main direction can be detected. This assumption allows us to reduce the extraction of the frequency information from two dimensions to one. Thus, the recognition of a peak along any arbitrary direction in the DFT image would be sufficient to provide the frequency information. However, in order to increase the reliability of the density estimation procedure, a more robust radial peak analysis technique was developed.

A total of 500 concentric circular rings, with equally spaced increasing radii, were considered in the DFT image. For each ring DFT magnitudes inside the ring were considered, their statistical distribution was derived, and the maximum value for each ring was determined. These 500 values of DFT magnitudes, one for each ring, were represented as a one dimensional "frequency signal," a representative example of which is shown in figure 4 .

This frequency signal represents the maximum DFT magnitude in each ring (vertical axis) as a function of the increasing radii of the rings (horizontal axis), which

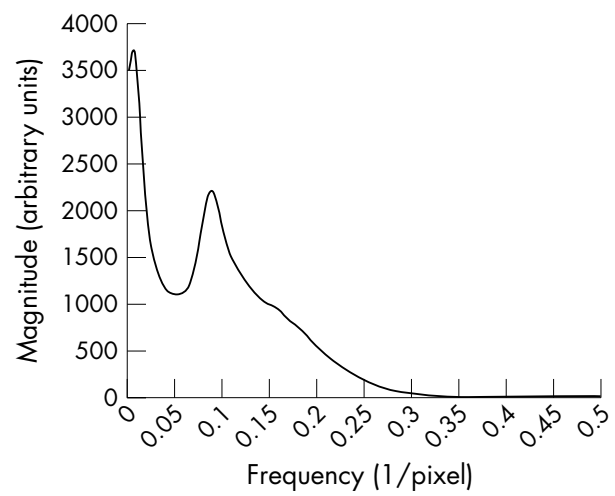

Figure 4 Course of the frequency signal, providing the cell density information (second peak). 
represent the spatial frequencies. By analysing this signal, two peaks are clearly identifiable, which indicate that two significant components are present in the frequency content of the image. The first peak (positioned at low frequency) is roughly related to the slow grey level variations in the image, most likely because of variation in image illumination and/or to endothelium layer folding; the second peak (positioned at higher frequency) is related to the frequency of the repetitive cell pattern, and thus to cell density. The second peak is thus the one we are interested in, and the spatial frequency value $f^{\prime},[1 /$ pixel], at which it is positioned contains the information about the cell density. The height of the peak does not contribute to the analysis, whereas the width of the peak represents the spreading of frequencies around the peak frequency, which is related to the degree of variation of density across the area under analysis. The cell density $d$ corresponding to the estimated spatial frequency $f^{\prime}$ is:

$$
d=\left(f^{\prime} c\right)^{2}
$$

where $c(\mathrm{pixel} / \mathrm{mm})$ is the linear spatial calibration factor of the instrument and camera system.

\section{Identification of non-processable images}

The algorithm described above is fully automatic and does not require any user intervention. In order to be reliably used in a clinical environment, however, it should be capable of assessing when the quality of the acquired image is not adequate to derive a correct estimation of cell density, so as to discard the image and not provide for it any density estimation. An analysis was performed on some images, which were judged of poor quality by eye bank experts because of the presence of wide unfocused regions, artefacts, or any other reason that prevented most of the cell borders to be clearly detected. For these reasons, these images had also been classified as unsuitable for manual cell counting by eye bank experts.

The application of the radial peak analysis to these images produced a frequency signal with more than two peaks (fig 5): four to five peaks were most often present in the signal. The presence of multiple peaks (that is, more than two) was thus correlated with the poor quality of the image. The algorithm described above was modified to recognise this particular situation and to provide a "non-processable image: no density available" warning.

The whole algorithm was implemented in the $\mathrm{C}++$ computer language and run on a personal computer.

\section{Clinical evaluation}

A clinical evaluation of the program was performed on 100 donor corneas. We chose to use as reference values, against

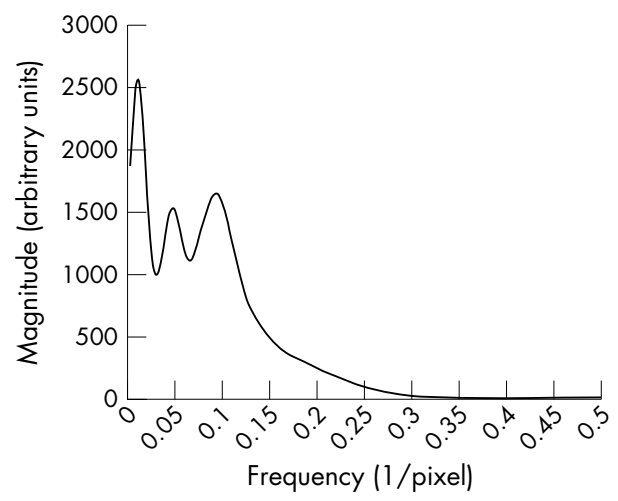

Figure 5 Course of the frequency signal for a non-processable image. which the automatic results were to be compared, the results of manual counting performed by a panel of eye bank experts. Two $200 \times$ digital images for each cornea (framed area $628 \mu \mathrm{m} \times 470 \mu \mathrm{m})$ were acquired from different central areas of the donor corneas (see Methods). Images with good cell visibility were used. By observing these latter images on a computer monitor and selecting a rectangular ROI (region of interest) occupying approximately $50 \%$ of the acquired image, visible cells were manually counted by three experienced investigators, for a total of 600 estimations. Only cells with clearly visible borders and entirely within the ROI, or touching one of two connected borders, were counted.

From the endothelium central zone of each of the same 100 donor corneas, several $100 \times$ digital images were also acquired. One image for each cornea, with the largest area of clearly visible cells, was chosen as the representative image for that cornea and automatic density estimation was performed by the proposed algorithm. The accuracy of these estimated densities was evaluated by direct comparison with the reference values.

Identification of non-processable images was also tested on the set of $100 \times$ images. For each cornea, all acquired $100 \times$ images were processed with the proposed algorithm and the capability of identification of poor quality images was evaluated.

\section{RESULTS}

The results of this clinical evaluation are reported in table 1 . Manual results are shown for each expert as the average between his/her estimated densities on the two $200 \times$ images for each cornea; the average between these experts' means, also reported, is used as reference density. Ranges of variation of manual densities by the three experts on the same corneas were computed: the average range of variation among single densities was 203 cells $/ \mathrm{mm}^{2}$ (8.6\%), with a maximum of 547 cells $/ \mathrm{mm}^{2}(22.8 \%)$.

The automatic densities reported are the values estimated by the program on the $100 \times$ representative image for each cornea. The average difference between automatic and reference manual densities (experts' average) was 14 cells/ $\mathrm{mm}^{2}(0.9 \%)$, with a standard deviation of $119 \mathrm{cells} / \mathrm{mm}^{2}$ $(5.1 \%)$, an average absolute difference of $92 \mathrm{cells} / \mathrm{mm}^{2}$ (3.9\%), and a maximum difference of 333 cells $/ \mathrm{mm}^{2}$ $(15.7 \%)$. In figure 6 a scatter plot of these densities is shown. To display the extent of agreement, a plot of difference $v$ mean for each pair of manual and automatic densities is shown in figure 7 , with three lines indicating the average difference and the $95 \%$ limits of agreement. ${ }^{18}$

The overall inter-rater reliability, ${ }^{19}$ obtained by an analysis of variance (ANOVA) including both the three human experts and the program, was 0.935. In order to statistically assess the agreement between the single human experts and between the manual reference (experts' average) and the program, ratios of estimated densities were computed. Their summary statistics are reported in table 2: the average of the ratio between manual reference and program is 1.009 , with a $95 \%$ confidence interval of 0.999 to 1.019 .

Finally, during the evaluation study a total of $393100 \times$ images on the 100 corneas were acquired, many of them purposely out of focus. The program was able to detect all the poor quality images and provide for them a "nonprocessable" warning, whereas in all the others the density was correctly estimated.

Automatic density estimation on a mid-range personal computer (Intel Pentium 4 CPU with 512 Mb RAM) required between 1 and 2 seconds of CPU time for each image. 
Table 1 Values of endothelial cell density in the 100 corneas of the evaluation set

\begin{tabular}{|c|c|c|c|c|c|c|c|c|c|}
\hline No & Expert 1 & Expert 2 & Expert 3 & $\begin{array}{l}\text { Manual } \\
\text { average }\end{array}$ & $\begin{array}{l}\text { Manual densities } \\
\text { range }\end{array}$ & $\begin{array}{l}\% \text { Manual } \\
\text { densities range }\end{array}$ & Automatic & Difference & \% Difference \\
\hline 1 & 2758 & 2614 & 2820 & 2731 & 338 & 12.4 & 2758 & 27 & 1.0 \\
\hline 2 & 2684 & 2647 & 2654 & 2661 & 216 & 8.1 & 2494 & -167 & -6.3 \\
\hline 3 & 2223 & 2186 & 2279 & 2229 & 133 & 6.0 & 2157 & -72 & -3.3 \\
\hline 4 & 2189 & 2078 & 2174 & 2147 & 212 & 9.9 & 2216 & 69 & 3.2 \\
\hline 5 & 2108 & 2070 & 2181 & 2119 & 193 & 9.1 & 2399 & 280 & 13.2 \\
\hline 6 & 2627 & 2618 & 2673 & 2639 & 104 & 3.9 & 2526 & -113 & -4.3 \\
\hline 7 & 2879 & 2910 & 2839 & 2876 & 243 & 8.5 & 2895 & 19 & 0.7 \\
\hline 8 & 2326 & 2399 & 2414 & 2379 & 180 & 7.6 & 2559 & 179 & 7.5 \\
\hline 9 & 2082 & 2076 & 2102 & 2087 & 239 & 11.5 & 2186 & 100 & 4.8 \\
\hline 10 & 2182 & 2261 & 2298 & 2247 & 235 & 10.5 & 2276 & 29 & 1.3 \\
\hline 11 & 1989 & 1977 & 2059 & 2008 & 123 & 6.1 & 2098 & 90 & 4.5 \\
\hline 12 & 2115 & 2048 & 2171 & 2111 & 205 & 9.7 & 2276 & 165 & 7.8 \\
\hline 13 & 1889 & 1821 & 2021 & 1910 & 271 & 14.2 & 2070 & 160 & 8.4 \\
\hline 14 & 1986 & 1866 & 1959 & 1937 & 241 & 12.4 & 2041 & 104 & 5.4 \\
\hline 15 & 2619 & 2560 & 2633 & 2604 & 183 & 7.0 & 2690 & 87 & 3.3 \\
\hline 16 & 2449 & 2243 & 2491 & 2394 & 547 & 22.8 & 2591 & 197 & 8.2 \\
\hline 17 & 1990 & 1843 & 1970 & 1934 & 204 & 10.5 & 1957 & 23 & 1.2 \\
\hline 18 & 2130 & 2044 & 2197 & 2123 & 246 & 11.6 & 2041 & -82 & -3.9 \\
\hline 19 & 2079 & 1986 & 2049 & 2038 & 136 & 6.7 & 2157 & 119 & 5.8 \\
\hline 20 & 2268 & 2222 & 2395 & 2295 & 239 & 10.4 & 2216 & -79 & -3.4 \\
\hline 21 & 2158 & 2138 & 2210 & 2168 & 149 & 6.9 & 2157 & -12 & -0.5 \\
\hline 22 & 2570 & 2554 & 2613 & 2579 & 185 & 7.2 & 2559 & -20 & -0.8 \\
\hline 23 & 2248 & 2219 & 2263 & 2243 & 282 & 12.6 & 2431 & 187 & 8.4 \\
\hline 24 & 2356 & 2323 & 2272 & 2317 & 207 & 8.9 & 2368 & 51 & 2.2 \\
\hline 25 & 2570 & 2532 & 2446 & 2516 & 269 & 10.7 & 2494 & -22 & -0.9 \\
\hline 26 & 2308 & 2220 & 2396 & 2308 & 352 & 15.3 & 2276 & -32 & -1.4 \\
\hline 27 & 2328 & 2158 & 2220 & 2235 & 224 & 10.0 & 2307 & 71 & 3.2 \\
\hline 28 & 2045 & 2032 & 2108 & 2062 & 124 & 6.0 & 2098 & 37 & 1.8 \\
\hline 29 & 2251 & 2146 & 2286 & 2227 & 195 & 8.8 & 2368 & 141 & 6.3 \\
\hline 30 & 1939 & 1945 & 2061 & 1981 & 372 & 18.8 & 2157 & 176 & 8.9 \\
\hline 31 & 2264 & 2267 & 2414 & 2315 & 207 & 8.9 & 2276 & -39 & -1.7 \\
\hline 32 & 1710 & 1650 & 1758 & 1706 & 126 & 7.4 & 1692 & -14 & -0.8 \\
\hline 33 & 1812 & 1813 & 2025 & 1883 & 228 & 12.1 & 2070 & 186 & 9.9 \\
\hline 34 & 2307 & 2152 & 2209 & 2223 & 202 & 9.1 & 2399 & 177 & 8.0 \\
\hline 35 & 2199 & 2072 & 2202 & 2157 & 210 & 9.7 & 2307 & 149 & 6.9 \\
\hline 36 & 2237 & 2020 & 2130 & 2129 & 280 & 13.2 & 2462 & 333 & 15.7 \\
\hline 37 & 2025 & 1980 & 2113 & 2039 & 183 & 9.0 & 2307 & 267 & 13.1 \\
\hline 38 & 2480 & 2447 & 2539 & 2488 & 325 & 13.1 & 2186 & -302 & -12.1 \\
\hline 39 & 2348 & 2268 & 2375 & 2330 & 260 & 11.2 & 2337 & 7 & 0.3 \\
\hline 40 & 3047 & 2945 & 2972 & 2988 & 128 & 4.3 & 2965 & -23 & -0.8 \\
\hline 41 & 2900 & 2875 & 2838 & 2871 & 223 & 7.8 & 2792 & -79 & -2.8 \\
\hline 42 & 2151 & 2083 & 2212 & 2148 & 192 & 8.9 & 2216 & 68 & 3.1 \\
\hline 43 & 2078 & 2025 & 2053 & 2052 & 124 & 6.0 & 2098 & 47 & 2.3 \\
\hline 44 & 2004 & 1931 & 2080 & 2005 & 284 & 14.2 & 2013 & 8 & 0.4 \\
\hline 45 & 2063 & 2078 & 2066 & 2069 & 125 & 6.0 & 2070 & 1 & 0.0 \\
\hline 46 & 2317 & 2275 & 2339 & 2310 & 117 & 5.1 & 2494 & 184 & 8.0 \\
\hline 47 & 2593 & 2423 & 2508 & 2508 & 201 & 8.0 & 2399 & -109 & -4.3 \\
\hline 48 & 1976 & 1995 & 2000 & 1990 & 126 & 6.3 & 1929 & -61 & -3.1 \\
\hline 49 & 2675 & 2588 & 2579 & 2614 & 312 & 11.9 & 2526 & -88 & -3.4 \\
\hline 50 & 2136 & 2012 & 2190 & 2112 & 201 & 9.5 & 2276 & 164 & 7.8 \\
\hline 51 & 2946 & 2798 & 2926 & 2890 & 201 & 7.0 & 2758 & -132 & -4.6 \\
\hline 52 & 2776 & 2745 & 2787 & 2769 & 155 & 5.6 & 2674 & -95 & -3.4 \\
\hline 53 & 2922 & 2806 & 2829 & 2852 & 326 & 11.4 & 2826 & -26 & -0.9 \\
\hline 54 & 2631 & 2656 & 2680 & 2655 & 151 & 5.7 & 2542 & -113 & -4.3 \\
\hline 55 & 2475 & 2472 & 2537 & 2494 & 158 & 6.3 & 2462 & -32 & -1.3 \\
\hline 56 & 2266 & 2295 & 2334 & 2298 & 113 & 4.9 & 2353 & 54 & 2.4 \\
\hline 57 & 2466 & 2444 & 2526 & 2479 & 132 & 5.3 & 2510 & 32 & 1.3 \\
\hline 58 & 2241 & 2234 & 2384 & 2286 & 242 & 10.6 & 2353 & 67 & 2.9 \\
\hline 59 & 2326 & 2327 & 2436 & 2363 & 232 & 9.8 & 2337 & -25 & -1.1 \\
\hline 60 & 2434 & 2425 & 2455 & 2438 & 223 & 9.1 & 2368 & -69 & -2.8 \\
\hline 61 & 2592 & 2553 & 2584 & 2576 & 234 & 9.1 & 2575 & -1 & 0.0 \\
\hline 62 & 2620 & 2650 & 2629 & 2633 & 304 & 11.5 & 2559 & -74 & -2.8 \\
\hline 63 & 2057 & 2090 & 2123 & 2090 & 118 & 5.6 & 2186 & 97 & 4.6 \\
\hline 64 & 2501 & 2517 & 2564 & 2527 & 93 & 3.7 & 2741 & 214 & 8.5 \\
\hline 65 & 2517 & 2513 & 2580 & 2536 & 130 & 5.1 & 2559 & 22 & 0.9 \\
\hline 66 & 2395 & 2389 & 2431 & 2405 & 110 & 4.6 & 2526 & 122 & 5.1 \\
\hline 67 & 2469 & 2482 & 2482 & 2477 & 66 & 2.7 & 2542 & 65 & 2.6 \\
\hline 68 & 2711 & 2580 & 2694 & 2661 & 230 & 8.6 & 2640 & -21 & -0.8 \\
\hline 69 & 3048 & 3027 & 3131 & 3068 & 187 & 6.1 & 3000 & -68 & -2.2 \\
\hline 70 & 3042 & 3087 & 3166 & 3098 & 219 & 7.1 & 3072 & -26 & -0.8 \\
\hline 71 & 2603 & 2562 & 2584 & 2583 & 83 & 3.2 & 2494 & -88 & -3.4 \\
\hline 72 & 2510 & 2506 & 2506 & 2507 & 70 & 2.8 & 2399 & -108 & -4.3 \\
\hline 73 & 2237 & 2233 & 2256 & 2242 & 202 & 9.0 & 2201 & -41 & -1.8 \\
\hline 74 & 2230 & 2188 & 2230 & 2216 & 78 & 3.5 & 2171 & -44 & -2.0 \\
\hline 75 & 2299 & 2419 & 2359 & 2359 & 255 & 10.8 & 2399 & 41 & 1.7 \\
\hline 76 & 2431 & 2448 & 2521 & 2467 & 125 & 5.1 & 2526 & 60 & 2.4 \\
\hline 77 & 2167 & 2207 & 2214 & 2196 & 355 & 16.2 & 2261 & 66 & 3.0 \\
\hline 78 & 1946 & 1926 & 2010 & 1961 & 159 & 8.1 & 1929 & -31 & -1.6 \\
\hline 79 & 1810 & 1899 & 1896 & 1868 & 235 & 12.6 & 1971 & 103 & 5.5 \\
\hline 80 & 2726 & 2621 & 2605 & 2651 & 397 & 15.0 & 2708 & 57 & 2.2 \\
\hline
\end{tabular}


Table 1 Continued

\begin{tabular}{|c|c|c|c|c|c|c|c|c|c|}
\hline No & Expert 1 & Expert 2 & Expert 3 & $\begin{array}{l}\text { Manual } \\
\text { average }\end{array}$ & $\begin{array}{l}\text { Manual densities } \\
\text { range }\end{array}$ & $\begin{array}{l}\% \text { Manual } \\
\text { densities range }\end{array}$ & Automatic & Difference & $\%$ Difference \\
\hline 81 & 2415 & 2371 & 2409 & 2398 & 111 & 4.6 & 2415 & 17 & 0.7 \\
\hline 82 & 3027 & 2812 & 2880 & 2906 & 399 & 13.7 & 2809 & -97 & -3.3 \\
\hline 83 & 3130 & 2950 & 3090 & 3057 & 210 & 6.9 & 2724 & -333 & -10.9 \\
\hline 84 & 2890 & 2775 & 2872 & 2845 & 255 & 9.0 & 2707 & -138 & -4.9 \\
\hline 85 & 2715 & 2542 & 2575 & 2610 & 285 & 10.9 & 2641 & 31 & 1.2 \\
\hline 86 & 2530 & 2509 & 2624 & 2554 & 187 & 7.3 & 2559 & 4 & 0.2 \\
\hline 87 & 2721 & 2696 & 2717 & 2711 & 107 & 3.9 & 2575 & -136 & -5.0 \\
\hline 88 & 2259 & 2274 & 2323 & 2285 & 154 & 6.7 & 2353 & 68 & 3.0 \\
\hline 89 & 2525 & 2463 & 2532 & 2506 & 115 & 4.6 & 2415 & -91 & -3.6 \\
\hline 90 & 2385 & 2433 & 2405 & 2407 & 138 & 5.7 & 2446 & 39 & 1.6 \\
\hline 91 & 2829 & 2730 & 2732 & 2764 & 152 & 5.5 & 2640 & -123 & -4.5 \\
\hline 92 & 1896 & 1917 & 1916 & 1910 & 254 & 13.3 & 1930 & 20 & 1.1 \\
\hline 93 & 2465 & 2417 & 2510 & 2464 & 259 & 10.5 & 2262 & -201 & -8.2 \\
\hline 94 & 2369 & 2398 & 2369 & 2379 & 107 & 4.5 & 2099 & -280 & -11.8 \\
\hline 95 & 2448 & 2383 & 2336 & 2389 & 143 & 6.0 & 2384 & -5 & -0.2 \\
\hline 96 & 2290 & 2297 & 2235 & 2274 & 143 & 6.3 & 2114 & -160 & -7.0 \\
\hline 97 & 2393 & 2420 & 2440 & 2418 & 104 & 4.3 & 2431 & 14 & 0.6 \\
\hline 98 & 2307 & 2280 & 2375 & 2321 & 169 & 7.3 & 2415 & 95 & 4.1 \\
\hline 99 & 2462 & 2519 & 2426 & 2469 & 122 & 4.9 & 2462 & -7 & -0.3 \\
\hline 100 & 2191 & 2181 & 2232 & 2201 & 365 & 16.6 & 2291 & 90 & 4.1 \\
\hline Mean & 2383 & 2341 & 2403 & 2375 & 203 & 8.6 & 2389 & 14 & 0.9 \\
\hline SD & 313 & 304 & 289 & 299 & 84 & 3.7 & 261 & 119 & 5.1 \\
\hline Min & 1710 & 1650 & 1758 & 1706 & 66 & 2.7 & 1692 & -333 & -12.1 \\
\hline Max & 3130 & 3087 & 3166 & 3098 & 547 & 22.8 & 3072 & 333 & 15.7 \\
\hline
\end{tabular}

For each cornea, manual densities were obtained by three experts in two $200 \times$ images and averaged for each expert ("Expert 1," "Expert 2," "Expert 3"). The grand average over all experts was computed ("Manual average") and the range of the six manual densities was also derived ("Manual densities range") and expressed as a percentage of the manual average ("\% Manual densities range"). Automatic densities were obtained for each cornea in one $100 \times$ image by using the proposed technique ("Automatic") and the difference from the manual average was computed ("Difference") and expressed also as a percentage of the manual average ("\% Difference").

\section{DISCUSSION}

We developed and implemented a new algorithm for the automatic estimation of corneal endothelium density in donor corneas at eye banks. Noting the regularity of the endothelial cell pattern, we adopted the Fourier analysis to extract the main frequency of this pattern, from which an estimate of cell density was derived.

An evaluation of the proposed system was performed to assess its clinical reliability. The main problem of this analysis was the lack of reference values, against which the automatic results were to be compared. We chose as such the results of the manual analysis independently performed by three clinical experts in two different central regions for each cornea. The resulting manual density values were, however, affected by many confounding factors: various types of errors that human experts perform during the counting procedure (inter-expert differences), and the extrapolation of cell count

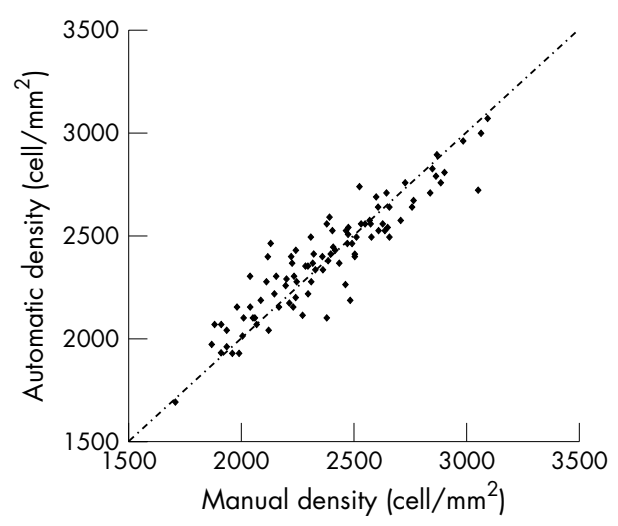

Figure 6 Scatter plot of manual $v$ automatic endothelium cell density estimates. done in a small ROI to the whole central region of the cornea, which is critical because of the physiological variation of endothelium density in different areas of the cornea. ${ }^{1620}$

It should also be noted that the manual counting procedure used here provided more accurate results than the conventional procedure adopted at most eye banks, where all cells inside a small $100 \mu \mathrm{m} \times 100 \mu \mathrm{m}$ graduated reticle are counted and the resulting value (between 15 and 40 cells) is then multiplied by 100 to obtain cell density as cells $/ \mathrm{mm}^{2}$. The variability of manual counts on the same cornea we obtained (mean 203 cells $/ \mathrm{mm}^{2}(8.6 \%)$, maximum 547 cells $\left./ \mathrm{mm}^{2}(22.8 \%)\right)$, which may be assumed as a statistical estimate of the error associated with the counted value, should thus be considered an optimistic lower limit to the ranges/errors commonly observed at eye banks. As a matter of fact, significantly larger variations were recently reported, with differences of up to $51 \%$ between technicians

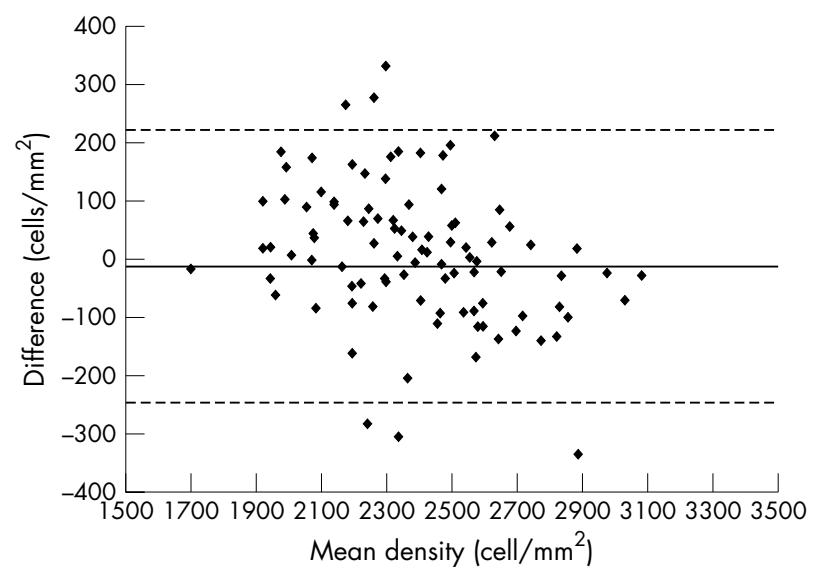

Figure 7 Scatter plot of difference $v$ mean for each pair of manual and automatic densities. The solid line shows the average difference, the broken lines show the $95 \%$ limits of agreement. ${ }^{18}$ 
Table 2 Summary statistics of the ratios of estimated densities between the three human experts ("Exp 1," "Exp 2," "Exp 3") and between the program ("Aut") and the experts' average ("Man")

\begin{tabular}{lllll}
\hline & Exp 2/Exp 1 & Exp 3/Exp 1 & Exp 3/Exp 2 & Aut/Man \\
\hline Mean & 0.983 & 1.010 & 1.028 & 1.009 \\
SD & 0.028 & 0.030 & 0.032 & 0.051 \\
Min & 0.903 & 0.949 & 0.963 & 0.879 \\
Max & 1.052 & 1.117 & 1.117 & 1.157 \\
$95 \% \mathrm{Cl}$ & 0.977 to 0.989 & 1.004 to 1.016 & 1.022 to 1.035 & 0.999 to 1.019 \\
\hline
\end{tabular}

For each ratio, mean, standard deviation (SD), minimum and maximum values, and $95 \%$ confidence interval are reported.

of the same eye bank and of $82 \%$ between technicians of different eye banks. ${ }^{21}$

Even if the density provided by this approach is only an estimation of the one yielded by a more accurate system, hypothetically able to correctly recognise the contour of each single cell, the results of the clinical evaluation showed a remarkably good agreement between automatic and manual densities: the average difference is less than $1 \%$ and the maximum absolute difference on all corneas is less than $16 \%$. These results are comparable with the ranges of variation of manual densities and much lower than those reported in other studies. ${ }^{21}$ The ratios between automatic densities and manual references have a very narrow 95\% confidence interval, including unity. These results statistically confirm the remarkably good agreement between automatic and manual densities. Obtaining the density values by analysing an endothelium area more than 100 times larger than the one conventionally used for manual count is definitely more robust against local variations of density and local artefacts of various origin. Moreover, since the density can be automatically estimated in a very short time, multiple estimations in different areas of the central region of cornea can be quickly performed and an average value that minimises possible biases can be obtained.

The proposed system is fully automatic. Only the selection of the images to be processed is done by the user, who can visually assess the quality of the image and avoid running the program if it is clearly out of focus or heavily corrupted by artefacts. However, the capability for an automatic program to detect by itself bad-that is, non-processable, images is certainly a desirable feature. The system proved to be quite robust against folds and other artefacts, which in most cases did not prevent it estimating a correct cell density. On the other hand, in some instances it appeared to be sensitive to out of focus images, where it exhibited additional peaks in the frequency signal. In order to recognise these specific situations, we developed a custom analysis, which proved able to identify all the non-processable images.

In summary, the proposed automatic system appears to be adequate to replace the much slower, more cumbersome, and error prone manual density evaluation of endothelium density. Since other proposed systems are at most semiautomatic and require considerable user interaction, this is the only viable fully automatic alternative to the manual procedure. The objective and fully reproducible results this system can provide may foster its adoption as a standardised procedure, through which corneas within and between eye banks can be reliably assessed and quantitatively compared.

\section{ACKNOWLEDGEMENTS}

This work was supported in part by Nidek Technologies, Italy. The authors wish to thank Dr R Rieger and Mrs C Jaeckel, Cornea Bank
Berlin, Charité, Germany, for their invaluable collaboration in the clinical evaluation. Preliminary reports were presented at the 2004 Conference of the European Eye Bank Association in Barcelona, Spain.

\section{Authors' affiliations}

A Ruggeri, E Grisan, Department of Information Engineering, University of Padova, Padova, Italy

J Jaroszewski, Cornea Bank Berlin, Charitè, Clinic of Ophthalmology, University School of Medicine Berlin, Campus Virchow-Klinikum, Berlin, Germany

\section{REFERENCES}

1 Bourne WM. Examination and photography of donor corneal endothelium. Arch Ophthalmol 1976;94:1799-800.

2 Lester JM, MacFarland JL, Bursell SE, et al. Automated morphometric analysis of corneal endothelial cells. Invest Ophthalmol Vis Sci 1981;20:407-10.

3 Hirst LW, Sterner RE, Grant DG. Automated analysis of wide-field specular photomicrographs. Cornea 1984;3:83-7.

4 Schultz RO, Matsuda M, Yee RW, et al. Corneal endothelium changes in type I and type II diabetes mellitus. Am J Ophthalmol 1984;98:401-10.

5 Hartmann C, Köditz W. Automated morphometric endothelial analysis combined with video specular microscopy. Cornea 1984/1985;3:155-67.

6 Matsuda M, Yee RW, Glasser DB, et al. Specular microscopic evaluation of donor corneal endothelium. Arch Ophthalmol Feb 1986;104:259-62.

7 Nishi O, Hanasaki K. Automated morphometry of corneal endothelial cell: use of video camera and video recorder. Br J Ophthalmol 1988;72:68-73.

8 Siertsema JV, Landesz M, Van den Brom H, et al. Automated video image morphometry of corneal endothelium. Doc Ophthalmol 1993;85:35-44.

9 Corkidi G, Marquez J, Usisima R, et al. Automated in vivo and online morphometry of human endothelium. Med Biol Eng Comput 1993;31:421-6.

10 Barisani-Asenbauer T, Baumgartner I, Grabner G, et al. Automated digital image analysis of organ culture preserved donor corneas. Ophthalmic Res 1993;25:94-9.

11 Sanchez-Marin FJ. Automatic segmentation of contours of corneal cells. Comput Biol Med 1999;29:243-58.

12 Reinhard T, Spelsberg H, Holzwarth D, et al. Wissensbasierte bildanalyse des endothels von hornhauttransplantaten. Klin Monatsbl Augenheilkd 1999:214:407-11.

13 Gain $P$, Thuret $G$, Gavet $Y$, et al. Automated tri-image analyser of stored corneal endothelium. Br J Ophthalmol 2002;86:801-8.

14 Masters BR. Characterization of corneal specular endothelial photomicrographs by their Fourier transforms. In: Juday R, ed. Digital and optical shape representation and pattern recognition-Proc SPIE 938, 1988:246-52.

15 Fitzke FW, Masters BR, Buckley RJ, et al. Fourier transform analysis of human corneal endothelial specular photomicrographs. Exp Eye Res 1997:65:205-14.

16 Amann J, Holley GP, Lee S, et al. Increased endothelial cell density in the paracentral and peripheral regions of the human cornea. Am J Ophthalmol 2003;135:584-90.

17 Gonzalez RC, Woods RE. Digital image processing. NJ: Prentice Hall, 2002.

18 Bland JM, Altman DG. Statistical methods for assessing agreement between two methods of clinical measurement. Lancet 1986;1:307-10.

19 Fleiss JL. The design and analysis of clinical experiments. New York: Wiley Interscience, 1986.

20 Waring GO, Bourne WM, Edelhauser HF. The corneal endothelium: normal and pathologic structure and function. Ophthalmology 1982;89:531-90.

21 Thuret G, Acquart S, Manissolle C, et al. Urgent need for normalization of corneal graft quality controls on French eye banks. In: Proceedings 16th EEBA Conference. Barcelona: European Eye Bank Association, 2004:25. 